\title{
El desempeño electoral de los partidos políticos provinciales en las elecciones seccionales 2014 en Ecuador
}

\section{The Electoral Performance of Provincial Parties in the 2014 Local and Provincial Elections in Ecuador}

Régis Dandoy

Recepción: 30 de julio de 2014

Aceptación: 9 de agosto de 2014

\section{Resumen}

Este artículo se enfoca en el desempeño electoral de los partidos de carácter provincial, tanto en elecciones provinciales (prefectos) como en elecciones cantonales (alcaldes) y observa hasta qué punto estas elecciones están nacionalizadas o provincializadas. El artículo concluye con tres hallazgos: primero, los partidos nacionales a menudo decidieron tener una estrategia provincial; segundo, los partidos nacionales decidieron crear una alianza electoral con un partido provincial para las elecciones locales, en lugar de con un partido local; tercero, las alianzas electorales entre un partido nacional y un partido provincial para las elecciones provinciales y locales probaron ser una estrategia exitosa.

Palabras claves: partidos provinciales, alianzas electorales, elecciones seccionales, provicialización de las elecciones, Ecuador.

\begin{abstract}
This article focuses on the electoral performance of provincial parties in both provincial (prefects) and cantonal (mayors) elections and observes in how far these elections are nationalised or provincialized. It concludes with three findings: first, national parties often decided to have a provincial strategy; second, national parties decided to create an electoral alliance with a provincial party for the local elections, rather than with a local party; and third, electoral alliances between a national party and a provincial party for the provincial and local elections were proved to be a successful strategy.
\end{abstract}

Keywords: provincial parties, electoral alliances, subnational elections, provincialization of elections, Ecuador. 


\section{Introducción}

U

na de las principales características del sistema de partidos en el Ecuador es la ausencia de partidos nacionales ${ }^{1}$ o en otras palabras, ausencia de partidos que compitan en todas las elecciones y por todo el territorio. Muchos de los más prominentes partidos políticos participan en elecciones en una determinada (geográficamente) región o en un limitado número de provincias. Algunos extractos de literatura en estudios electorales se han concentrado en el proceso de "nacionalización" de las elecciones en Ecuador (ver por ejemplo Polga-Hecimovich, 2013) pero la mayor parte de estos estudios se concentran únicamente en las elecciones nacionales y dejan de lado las elecciones subnacionales.

El Ecuador es un país con un sistema descentralizado, donde las elecciones provinciales, cantonales y parroquiales son de gran importancia (sin mencionar las elecciones de los parlamentarios andinos). Además, el sistema de partidos no se puede reducir a los partidos nacionales y su importancia en los resultados electorales. La mayoría de partidos tienen aspiraciones electorales a nivel cantonal o provincial, como partidos independientes o en alianzas electorales con otros partidos (algunas veces partidos nacionales).

Este artículo observa cómo los partidos de carácter nacional -directa o indirectamente- ganaron las elecciones en el 2014, en la mayor parte de las provincias y cantones y, que hasta ahora, acumularon la mayoría de votos. Pero ¿qué sucede con los resultados y estrategias de los candidatos y los partidos provinciales? ¿Están ellos ausentes completamente del proceso electoral? Y ¿cómo lograron obtener importantes cuotas de los votos, incluso en competencia con los partidos nacionales?

El proceso electoral es posible entender haciendo referencia a dos perspectivas extremas. La primera implica que las elecciones provinciales son completamente provincializadas. Por definición, las elecciones

1 En este artículo, se utilizará indiferenciadamente "partido" para todos los partidos y movimientos políticos. 
provinciales son un fenómeno provincial y deberían ser la preocupación principal de los partidos y los candidatos a nivel provincial. La campaña electoral debería concentrarse en los problemas provinciales y hacer publicidad en los medios de comunicación provincial. La segunda implica que las elecciones provinciales son completamente nacionalizadas. Únicamente los partidos y los candidatos a nivel nacional participan en estas elecciones, y los resultados electorales están completamente subordinados a cuestiones nacionales, el desempeño de los partidos de gobierno a nivel nacional, y al calendario electoral nacional.

Este artículo particularmente se enfoca en analizar el desempeño electoral de los partidos de carácter provincial, tanto en elecciones provinciales (prefectos) como en elecciones cantonales (alcaldes) y observa hasta qué punto estas elecciones están nacionalizadas o provincializadas.

\section{Partidos nacionales y provinciales}

La definición de partidos provinciales no es una tarea fácil, además es una definición paralelamente dependiente de la definición de partidos nacionales. Para comprender lo que es un partido provincial, es preciso primeramente determinar el alcance y las características de los partidos nacionales.

Existen básicamente tres formas para definir a un partido nacional, que son: legal, política y electoral.

Basado en la perspectiva legal, la primera definición de un partido nacional implica el reconocimiento formal por parte del Estado. En Ecuador, los artículos 310 y 311 de la Ley Orgánica Electoral y de Organizaciones Políticas de la República del Ecuador, Código de la Democracia, define a los partidos políticos nacionales ${ }^{2}$. El Consejo Nacional Electoral (CNE)

2 Artículo 310.- Los partidos políticos serán de carácter nacional, se regirán por sus principios y estatutos, propondrán un programa de gobierno y mantendrán el registro de sus afiliados. El carácter de los movimientos políticos se determinará en su respectivo régimen orgánico y podrán corresponder a cualquier nivel de gobierno o a la circunscripción especial del exterior. Artículo 311.- Las denominaciones "partido político", "movimiento político" y "alianza" se reservan exclusivamente a aquellos inscritos como tales en el Registro Nacional de organizaciones políticas que para tal efecto mantiene el Consejo Nacional Electoral. 
reconoció doce (12) partidos de alcance nacional: Movimiento Popular Democrático (MPD), Partido Roldosista Ecuatoriano (PRE), Partido Socialista-Frente Amplio (PS-FA), Partido Renovador Institucional Acción Nacional (PRIAN), Partido Social Cristiano (PSC), Partido Sociedad Patriótica 21 de enero (PSP), Partido Avanza, Movimiento Ruptura 25, Sociedad Unida Más Acción (SUMA), Movimiento Creando Oportunidades (CREO), Movimiento Unidad Plurinacional Pachakutik (MUPP), y Movimiento Alianza País (MPAIS), Patria Altiva I Soberana (AP). El problema principal de esta definición, en el caso de las elecciones seccionales 2014, es que no todos los partidos nacionales participaron en dichas elecciones, tal es el caso del Movimiento Ruptura 25.

La segunda definición tiene un sustento político, es decir si un partido está presente en la Asamblea Nacional. Según esta definición, Ecuador ha presenciado desde las elecciones de 2013, a no menos de once (11) partidos nacionales participando en la Asamblea: AP, CREO, PSC, MPD/MUPP, Avanza, PRE, SUMA, ARE, IDC y MPCG. Sin embargo, algunos partidos provinciales también participaron en las elecciones nacionales, y por tanto, están representados en la Asamblea Nacional, este es el caso de Integración Democrática del Carchi (IDC).

Una tercera definición de partido nacional se sustenta en el criterio electoral, es decir simplemente si un partido participó en elecciones (nacionales, provinciales o locales) en todo el país. Ahora, puede existir un criterio diferente basado en el número de provincias que es necesario alcanzar para ser considerado un partido nacional: participar en elecciones en todas las provincias, en la mayoría de provincias o al menos en dos provincias.

En el marco de este artículo, se define al partido nacional como un partido que presenta candidatos para las elecciones a nivel provincial en al menos dos provincias. De igual manera, un partido provincial se define como un partido que participa en las elecciones provinciales en solo una provincia, o como un partido que participa en al menos dos cantones de una provincia.

Los partidos provinciales pueden también ser identificados con base a su nominación; es decir, si este se refiere a la provincia o al grupo de 
cantones en un solo nombre del partido; sin embargo, el objetivo de este artículo es analizar el desempeño electoral y las estrategias de estos partidos y no de sus ideologías, este artículo se basa en la definición de dichos partidos. Finalmente, un partido local es definido como un partido que participa en las elecciones locales en un solo cantón. Por definición, no existen partidos locales dentro de las elecciones provinciales.

Con ocasión de las elecciones provinciales de febrero de 2014 en Ecuador, no menos de ocho (8) partidos presentaron un candidato en una sola provincia. Este es por ejemplo el caso de IDC en la provincia del Carchi, o de Unidos por Pastaza en la provincial de Pastaza. Además, sesenta (60) partidos presentaron candidatos en al menos dos cantones en una provincia para las elecciones locales. Estos partidos son por tanto, considerados como partidos provinciales. Por el contrario, el número de partidos que participaron en las elecciones seccionales de 2014 en un solo cantón, es menor: cuarenta y seis (46) de estos pueden ser considerados como partidos locales.

En comparación con el potencial número de partidos nacionales -al cual se añaden las organizaciones políticas provinciales y localesse observa que el número promedio de candidatos por cantón es más bien bajo (5,45 partidos) lo que significa que la mayor parte de estos partidos (nacionales) decidieron no participar en varios cantones. De hecho, los partidos políticos pueden elegir entre diferentes estrategias (para mayor información ver: Dandoy, 2014).

Primero, estos partidos pueden presentar un candidato -bajo el propio nombre del partido o en alianza- en todos los cantones y provincias (como MPAIS) o en una gran mayoría de los cantones. Por ejemplo, Avanza y CREO estuvieron presentes en los cantones en un 79,09\% y $74,09 \%$ respectivamente. CREO tuvo candidatos en diecinueve (19) provincias mientras que Avanza estuvo presente en trece (13) de ellas. Algunos partidos nacionales participaron en las elecciones para alcaldes en la mayoría de los cantones, como por ejemplo: PS-FA y SUMA $(51,82 \%$ de los cantones), PSP $(50,91 \%)$, MUPP $(41,81 \%)$ y MPD (39,55\%). Otros optaron por presentar un candidato en menos cantones, como PSC y PRE 
(31,36\%) o PRIAN (21,36\%). El PSC y PRIAN tuvieron candidatos en siete (7) y seis (6) provincias respectivamente.

Segundo, los partidos pueden presentar un candidato para las elecciones de alcalde y otro para las elecciones para prefecto con el nombre del propio partido o en alianza. La mayoría de candidatos para estas elecciones fueron apoyados por un solo partido (es decir, solo un partido en la papeleta, junto al nombre del candidato). Sin embargo, algunos partidos políticos decidieron unir sus fuerzas (con frecuencia contra el candidato dominante o el acalde o prefecto titular) y conjuntamente apoyar un solo candidato.

Esta unificación entre partidos políticos toma la forma electoral conocida como alianza, que algunas veces reúne a partidos muy diferentes, no solamente en términos de ideología sino también en términos de alcance territorial; no es extraño observar una alianza entre un partido nacional con un partido provincial o una alianza entre un partido provincial con un partido local. En 2014, no menos del 26,72\% de todos los candidatos para elecciones de prefecto y $22,65 \%$ de todos los candidatos para elecciones de alcalde fueron auspiciados por una alianza electoral (ver más información debajo).

Del mismo modo, las estrategias adoptadas por los partidos nacionales para presentar candidatos en todos los cantones o solo en un limitado número de cantones, algunos partidos nacionales escogieron ser parte de una alianza electoral, mientras otros prefirieron participar en las elecciones con su propio nombre y membrete. Algunos partidos participaron en las elecciones principalmente en alianzas. Este es el caso del PSC (84,06\% de sus candidatos estuvieron en una alianza electoral) y el PRIAN (68,09\%). En otros casos, el número de candidatos que participaron en las elecciones compitieron bajo el nombre del partido. Este es el caso de PSP (46,43\%), AP (42,73\%), PS-FA (21,05\%) o Avanza (12,64\%).

Desde una perspectiva metodológica, el desempeño electoral de los partidos que componen estas alianzas es calculado sobre la proporción de los votos obtenidos por cada partido político dentro de esa alianza. Por ejemplo, el número de votos obtenido por una alianza de dos partidos será dividido en partes iguales entre los dos partidos; el número de 
votos obtenidos por una alianza de tres partidos será dividido en partes iguales entre los tres partidos, etc.

En suma, estas son estrategias diferentes -es decir para presentar un candidato por su propia cuenta o en alianza con otros partidos y presentar un candidato a todas las elecciones o solo en las circunscripciones claves- tiene un claro impacto en los resultados electorales.

\section{Resultados electorales de los partidos provinciales}

Como era de esperarse, se observó una importante nacionalización en las elecciones seccionales (provinciales y locales) en Ecuador. La gran mayoría de los candidatos que participaron en las elecciones locales y provinciales pertenecen a los partidos nacionales. El 68,53\% y el $66,38 \%$ de los candidatos a alcaldes y prefectos a elecciones respectivamente, fueron candidatos que estaban directamente asociados con un partido nacional (sin mencionar aquellos que están indirectamente unidos a esos partidos a través de varias alianzas electorales).

Incluso si la campaña electoral estaba supuestamente concentrada en cuestiones cantonales y provinciales, muchos de los actores tuvieron una estrategia de campaña a nivel nacional. Por ejemplo, varios partidos publicitaron las fotografías de candidatos locales y provinciales junto con el líder nacional del partido. En particular, la estrategia del partido de gobierno, AP fue dar un "sabor" nacional a las elecciones locales y provinciales, un año después de la victoria de este partido en la elecciones generales de febrero de 2013 .

A continuación, se analizará el desempeño de los partidos provinciales en las elecciones seccionales de febrero 2014 basado en tres perspectivas diferentes: los candidatos apoyados por estos partidos, el porcentaje (cuota) de votos obtenidos y finalmente los prefectos y alcaldes electos. 


\section{Elecciones para prefecto}

Se observa que veintiocho (28) candidatos provinciales participaron en las veintitrés (23) elecciones provinciales, lo que significa que, en promedio, hubo más de un candidato provincial por cada provincia. Como se menciona previamente, las elecciones provinciales están dominadas por candidatos y partidos nacionales (directamente representa el $66,38 \%$ e indirectamente el $92,24 \%$ de todos los candidatos).

Entre los 28 candidatos provinciales, solo ocho (8) de ellos participaron en las elecciones provinciales a través de un partido único, y un candidato - en la provincia de Azuay - participó para las elecciones a la prefectura a través de una alianza de dos partidos provinciales. Todos los otros candidatos provinciales fueron respaldados por alianzas electorales que contenían al menos un partido nacional.

Tabla 1.

Resultados electorales (prefectos 2014)

\begin{tabular}{c|c|c|c|c|c|c}
\hline \multirow{2}{*}{} & \multicolumn{2}{|c|}{ Candidatos } & \multicolumn{2}{c|}{ Votos } & \multicolumn{2}{c}{ Prefectos } \\
\cline { 2 - 7 } & $\mathrm{N}$ & $\%$ & $\mathrm{~N}$ & $\%$ & $\mathrm{~N}$ & $\%$ \\
\hline Partido Nacional & 77 & $66,38 \%$ & 3870840 & $46,15 \%$ & 12 & $52,17 \%$ \\
\hline Partido Provincial & 8 & $6,90 \%$ & 165395 & $1,97 \%$ & 1 & $4,35 \%$ \\
\hline Alianza & 31 & $26,72 \%$ & 4351760 & $51,88 \%$ & 10 & $43,48 \%$ \\
\hline Total & 116 & $100 \%$ & 8387995 & $100 \%$ & 23 & $100 \%$ \\
\hline
\end{tabular}

Fuente: Consejo Nacional Electoral (2014)

En la mayoría de los casos, estas alianzas electorales unieron un partido provincial con un partido nacional. Pero, en dos casos, fue una alianza entre dos partidos nacionales y un partido provincial. Esto es así para la alianza entre APAIS, PS-FA Y MIOSA en la provincia de Bolívar y la alianza entre PSP, CREO, y MIFA en Morona Santiago. En un caso, se trata de una alianza entre tres partidos nacionales y un partido provincial: la alianza entre CREO, PRE, Avanza and MOA en Orellana. Finalmente, un candidato de Santo Domingo estaba apoyado por la 
alianza de no menos de cinco partidos: tres nacionales (PSP, PRIAN, y MUPP) y dos partidos provinciales (A-TSA y MPSSD).

Desagregando las alianzas electorales de los partidos nacionales con los partidos provinciales, se puede observar que algunos partidos nacionales tienden a favorecer a este tipo de alianzas mientras otras prefieren presentarse solo o crear alianzas solamente con partidos nacionales (ver tabla 2). Existen diferentes estrategias en juego. Por ejemplo, APAIS presentó un candidato junto con un partido provincial en no menos que siete provincias. A este partido le sigue Avanza y CREO (cada uno con cuatro alianzas con un partido provincial). Por otro lado, el MPD no presentó un candidato junto con un partido provincial.

Tabla 2.

Número de alianzas electorales entre partidos nacionales y provinciales (por partido nacional)

\begin{tabular}{c|c|c}
\hline Partido & Elecciones de Prefectos & Elecciones de Alcaldes \\
\hline PSP & 2 & 12 \\
\hline PSC & 3 & 36 \\
\hline PRIAN & 1 & 0 \\
\hline Avanza & 4 & 6 \\
\hline PRE & 1 & 3 \\
\hline MPD & 0 & 7 \\
\hline PS-FA & 1 & 7 \\
\hline MUPP & 1 & 0 \\
\hline CREO & 4 & 23 \\
\hline SUMA & 1 & 12 \\
\hline APAIS & 7 & 70 \\
\hline
\end{tabular}

Nota: La suma de todas las alianzas electorales en esta tabla es mayor al número global de alianzas ya que, varios partidos nacionales pueden estar juntos en la misma alianza con un partido provincial.

En términos de número de votos obtenidos por los diferentes candidatos, se observa que los partidos nacionales ya no dominan las elecciones provinciales (ver tabla 1). Cuando un partido compite solo en elecciones, los partidos nacionales lograron solo obtener $46,15 \%$, porcentaje que es mucho menor a la proporción de candidatos que estos partidos 
presentaron (66,38\%). La categoría de partidos que realmente alcanzan el mayor número de votos son las alianzas electorales, estas obtienen no menos del $51,88 \%$ de los votos en las elecciones seccionales de febrero de 2014. Comparado con el bajo número de candidatos que fueron respaldados por dichas alianzas, estos partidos pueden ser considerados como ganadores de estas elecciones. Por el contrario, cuando los partidos provinciales participan solos en elecciones, estos no atraen muchos votos (menos del 2\% de los votos).

Como se observa anteriormente, algunos partidos provinciales decidieron crear alianzas electorales con partidos nacionales o con otros partidos provinciales. Generalmente, estas alianzas se desempeñan bien (ver tabla 3). De dieciocho (18) alianzas entre un partido provincial y al menos un partido nacional alcanzaron el $18,15 \%$ de los votos, y la alianza entre dos partidos provinciales obtuvo 2,67\% de los votos. En ambos casos las alianzas se desempeñaron proporcionalmente mejor que la cantidad de candidatos que estas apoyaron. La única (pequeña) excepción es la gran alianza entre tres partidos nacionales y dos provinciales.

Tabla 3.

Partidos provinciales (prefectos 2014)

\begin{tabular}{c|c|c|c|c|c|c}
\hline & \multicolumn{2}{|c|}{ Candidatos } & \multicolumn{2}{c|}{ Votos } & \multicolumn{2}{c}{ Prefectos } \\
\cline { 2 - 7 } & $\%$ & $\mathrm{~N}$ & $\mathrm{~N}$ & $\%$ & $\mathrm{~N}$ & $\%$ \\
\hline Un solo partido & 8 & $6,90 \%$ & 165395 & 1,97 & 1 & 4,35 \\
\hline Alianza con un partido nacional & 18 & $15,52 \%$ & 1522666 & 18,15 & 5 & 21,74 \\
\hline Alianza con un partido provincial & 1 & $0,86 \%$ & 224151 & 2,67 & 1 & 4,35 \\
\hline $\begin{array}{c}\text { Alianza con un partido nacional } \\
\text { y un provincial }\end{array}$ & 1 & $0,86 \%$ & 9224 & 0,11 & 0 & 0,00 \\
\hline Total partidos provinciales & $\mathbf{2 8}$ & $\mathbf{2 4 , 1 4 \%}$ & $\mathbf{1 9 2 1 4 3 5}$ & $\mathbf{2 2 , 9 1}$ & 7 & $\mathbf{3 0 , 4 3}$ \\
\hline Total de todos los partidos & $\mathbf{1 1 6}$ & $\mathbf{1 0 0} \%$ & $\mathbf{8 3 8 7 9 9 5}$ & $\mathbf{1 0 0}$ & $\mathbf{2 3}$ & $\mathbf{1 0 0}$ \\
\hline
\end{tabular}

Fuente: Consejo Nacional Electoral (2014)

Finalmente, este artículo analiza los candidatos electos. Como se esperaba, la mayor parte de ellos pertenece al partido nacional (12 prefectos 
electos) o a una alianza electoral (10 prefectos electos). En una provincia, el prefecto elegido representa al partido provincial. Este es el caso de Guillermo Herrera (IDC) que logró el 71,91\% de los votos en la provincia del Carchi. Entre otros casos exitosos de alianzas electorales, no menos de cinco (5) alianzas entre partidos nacionales y provinciales condujeron a elegir un candidato, por ejemplo en la provincia del Guayas, Imbabura, Loja, Manabí y Santa Elena. La alianza entre dos partidos provinciales en Azuay también condujo el triunfo de Paúl Ernesto Carrasco Carpio (PDR - MI - Alianza Participa con Igualdad) con el $57,08 \%$ de los votos.

\section{Elecciones para alcalde}

Las elecciones locales en Ecuador no son locales. Los candidatos auspiciados por listas locales participando individualmente representan solo el 3,83\% de todos los candidatos para las elecciones de alcaldes (ver tabla 4). De la misma manera que para las elecciones provinciales, las elecciones locales son dominadas por los partidos nacionales $(68,53 \%$ de todos los candidatos), seguido por las alianzas electorales. Los candidatos que pertenecen a un partido provincial participando individualmente representan solo el 5\% de los candidatos.

Esta observación es confirmada por el análisis de los resultados electorales. Las listas locales participando individualmente logran obtener el 1,98\% y cinco alcaldes electos (en los cantones de San Miguel, Santa Rosa, Espíndola, Zapotillo y Cevallos). Adicionalmente, solo un candidato logra ser electo apoyado por una alianza electoral entre dos partidos locales (Gustavo Silva, en el cantón de Mera, en Pastaza con el $37,45 \%$ de los votos $)^{3}$.

3 Para mayor información sobre el desempeño de los partidos locales en las elecciones para alcaldes, ver Dandoy, 2014. 
Tabla 4.

Resultados electorales

\begin{tabular}{c|c|c|c|c|c|c}
\hline \multirow{2}{*}{} & \multicolumn{2}{|c|}{ Candidatos } & \multicolumn{2}{c|}{ Votos } & \multicolumn{2}{c}{ Alcaldes } \\
\cline { 2 - 7 } & $\mathrm{N}$ & $\%$ & $\mathrm{~N}$ & $\%$ & $\mathrm{~N}$ & $\%$ \\
\hline Partido nacional & 823 & $68,53 \%$ & 3944629 & $45,88 \%$ & 138 & $62,44 \%$ \\
\hline Partido provincial & 60 & $5,00 \%$ & 299923 & $3,49 \%$ & 12 & $5,43 \%$ \\
\hline Partido local & 46 & $3,83 \%$ & 169985 & $1,98 \%$ & 5 & $2,26 \%$ \\
\hline Alianza & 272 & $22,65 \%$ & 4182821 & $48,65 \%$ & 66 & $29,86 \%$ \\
\hline Total & 1201 & $\mathbf{1 0 0} \%$ & $\mathbf{8 5 9 7 3 5 8}$ & $\mathbf{1 0 0 \%}$ & $\mathbf{2 2 1}$ & $\mathbf{1 0 0 \%}$ \\
\hline
\end{tabular}

Fuente: Consejo Nacional Electoral (2014)

Incluso si los partidos provinciales que participan individualmente presentan solo 60 candidatos, no menos que 230 candidatos fueron directamente o indirectamente apoyados por un partido provincial (ver tabla 5). La mayor parte de ellos (128) pertenecen a una alianza electoral entre un partido nacional y un partido provincial, al cual se podría añadir los 28 candidatos apoyados por una alianza electoral entre al menos un partido nacional y al menos dos partidos provinciales. Solamente, trece (13) candidatos son apoyados por una alianza electoral entre un partido provincial mientras solo uno fue respaldado por una alianza electoral entre un partido provincial y un partido local (Roberto Osejo en San Pedro de Huaca, provincia del Carchi).

En cuanto a los votos, estos candidatos provinciales obtuvieron el $44,81 \%$ de los votos a nivel nacional. Para los partidos provinciales, la estrategia de participar en las elecciones por su cuenta no fue muy exitosa: con un número relativamente pequeño de los candidatos provinciales $(5 \%)$ se eligió un mayor número de alcaldes $(5,43 \%)$ pero con una menor cuota de los votos (3,49\%).

Por el contrario, la estrategia de crear alianzas electorales con otros partidos ha demostrado ser exitosa. Las alianzas entre un partido provincial y al menos un partido nacional lograron obtener el 19,11\% de los votos (y el 15,11\% de alcaldes electos). Esta cifra relativamente alta de los votos obtenidos por alianzas entre un partido provincial y un 
partido nacional se explica por el hecho de que varios candidatos de los cantones más grandes recibieron el apoyo de este tipo de alianzas. Este es el caso de tres (3) de los más destacados candidatos para las elecciones de alcalde: Jaime Nebot (alianza entre PSC y Madera de Guerrero) en Guayaquil con 841901 votos, Mauricio Rodas (alianza entre SUMA y VIVE) en Quito con 823869 votos en Quito y Viviana Bonilla (alianza entre APAIS y Movimiento Centro Democrático) con 554337 votos en Guayaquil.

Los otros tipos de alianza que involucran a un partido provincial consiguieron obtener una significativa cantidad de votos, como es el caso de las alianzas entre dos partidos provinciales (1,93\% de los votos y tres alcaldes electos), las alianzas entre al menos un partido nacional y dos partidos provinciales (cinco alcaldes electos). El único candidato que compitió mediante una alianza entre un partido provincial y un partido local logró el segundo lugar en San Pedro de Huaca, confirmando el bajo grado de localismo en las elecciones locales. En suma, los 230 candidatos que fueron directa o indirectamente apoyados por un partido provincial obtuvieron $24,9 \%$ de los votos y $24,43 \%$ de alcaldes electos.

Tabla 5.

Partidos provinciales (alcaldes 2014)

\begin{tabular}{c|c|c|c|c|c|c}
\hline & \multicolumn{2}{|c|}{ Candidatos } & \multicolumn{2}{c|}{ Votos } & \multicolumn{2}{c}{ Alcaldes } \\
\cline { 2 - 8 } & $\mathrm{N}$ & $\%$ & $\mathrm{~N}$ & $\%$ & $\mathrm{~N}$ & $\%$ \\
\hline Un solo partido & 60 & 5,00 & 299923 & 3,49 & 12 & 5,43 \\
\hline Alianza con un partido nacional & 128 & 10,66 & 1643230 & 19,11 & 34 & 15,38 \\
\hline Alianza con un partido provincial & 13 & 1,08 & 165597 & 1,93 & 3 & 1,36 \\
\hline $\begin{array}{c}\text { Alianza con un partido nacional y } \\
\text { provincial }\end{array}$ & 28 & 2,33 & 30747 & 0,36 & 5 & 2,26 \\
\hline Alianza con un partido local & 1 & 0,08 & 1217 & 0,01 & 0 & 0,00 \\
\hline Total de partidos provinciales & 230 & $\mathbf{1 9 , 1 5}$ & $\mathbf{2 1 4 0 7 1 4}$ & $\mathbf{2 4 , 9 0}$ & $\mathbf{5 4}$ & $\mathbf{2 4 , 4 3}$ \\
\hline Total de los partidos & $\mathbf{1 2 0 1}$ & $\mathbf{1 0 0}$ & $\mathbf{8 5 9 7 3 5 8}$ & $\mathbf{1 0 0}$ & $\mathbf{2 2 1}$ & $\mathbf{1 0 0}$ \\
\hline
\end{tabular}

Fuente: Consejo Nacional Electoral (2014) 


\section{Conclusión}

Se ha podido observar las diferencias significantes entre las elecciones para alcalde y prefectos. Primero, los partidos nacionales no se desempeñan de la misma manera. Por ejemplo, APAIS obtuvo $26,25 \%$ de los votos en las elecciones para alcalde en todo el país, mientras que obtuvo el 34,45\% en las elecciones para prefectos (Dandoy, 2014). Segundo, puede parecer lógico que los partidos provinciales se desempeñan bien en las elecciones provinciales (22,91\% de los votos), es sorprendente que estos se desempeñen mejor en las elecciones locales $(24,90 \%$, es decir aproximadamente un cuarto de los votos).

Los partidos provinciales presentaron más candidatos que el número total de provincias y cantones, esto significa que hubo, en promedio, al menos un candidato provincial (o un candidato apoyado por un partido provincial) en cada provincia y en cada cantón. La mayoría de estos candidatos provinciales estuvieron respaldados por una alianza electoral entre un partido nacional y un partido provincial.

Ser un candidato respaldado por una alianza electoral fue la estrategia ganadora. De 221 cantones, estos candidatos ganaron en 65 de ellos, y obtuvieron más de cuatro (4) millones de votos. Ahora, esta cifra no es sorprendente ya que las alianzas electorales principalmente fueron observadas en los cantones por un gran número de votantes. En tres de las ciudades más grandes del país (Guayaquil, Quito y Cuenca), el candidato ganador compitió a través de una alianza electoral.

Además, se observa que existen diferentes tipos de alianzas: estas pueden unir dos partidos nacionales; un partido nacional y un partido provincial; uno nacional y uno local; dos partidos provinciales; uno provincial y un partido local; o incluso dos partidos locales. Entre estos tipos de alianzas, la más exitosa fue sin duda alguna las alianzas entre al menos un partido nacional. Las alianzas entre un partido nacional y un partido provincial ganaron en no menos de cinco provincias y 34 cantones y probaron ser también bastante exitosas. Las alianzas entre dos partidos provinciales ganaron las elecciones en una provincia y tres cantones. 
Las elecciones locales y provinciales en Ecuador están dominadas por los partidos nacionales. Sin embargo, aparte de la importancia de los actores y cuestiones nacionales también se observa claramente la "provincialización" de las elecciones de febrero de 2014. Estudios anteriores enfatizan que esta provincialización toma la forma de concentración de la cuota de votos que alcanza un partido en algunas provincias, mientras este partido se desempeña peor en otras (Pachano 2004, 2007). Pero el proceso de provincialización de las elecciones en Ecuador puede tomar otras formas que la forma provincial de concentración de los votos.

Primero, los partidos nacionales a menudo deciden tener una estrategia provincial. Con excepción de APAIS, todos los partidos nacionales deciden competir, por si solos o en alianzas electorales, para las elecciones de prefectos en algunas provincias y no competir en otras. Por ejemplo, Avanza y MUPP presentaron candidatos en 13 y 12 provincias respectivamente. Adicionalmente, los partidos nacionales también decidieron competir en las elecciones locales basados en el mismo tipo de estrategia en todos los cantones y provincias.

Los candidatos o su participación en alianzas electorales para las elecciones locales son con frecuencia concentrados en algunas provincias. Por ejemplo, la alianza electoral conformada por el partido nacional CRE, PSP y PRIAN en ocho de los nueve cantones de la provincia de Tungurahua. Esta alianza no está presente en ninguno de los cantones fuera de esta provincia. En la provincial de Bolívar, APAIS, PS-FA y un partido provincial decidieron participar juntos en las elecciones a través de una alianza con el nombre de "Integración Bolivarense", una alianza que está presente en todos los cantones de Bolívar pero en ningún otro cantón fuera de esta provincia.

Segundo, puede parecer lógico que los partidos nacionales decidan construir alianzas electorales con partidos provinciales para las elecciones provinciales. Más sorprendente es el hecho de que los partidos nacionales pueden decidir crear una alianza electoral con un partido provincial para las elecciones locales, en lugar de hacerlo con un par- 
tido local. Este tipo de alianzas electorales ocurrió en 128 casos, que representan el 10,66\% de todos los candidatos para todos los cantones.

Estas alianzas son mucho más frecuentes que las alianzas entre un partido nacional y un partido local, lo cual ocurrió solo doce (12) veces (aproximadamente el 1\% de todos los candidatos). Como se menciona previamente, los partidos nacionales tienden a adoptar la misma alianza electoral en todos los cantones de una provincia. Los ejemplos que se puede mencionar son las alianzas de PSC con Madera de Guerrero en los cantones de la provincia de Guayas, o la alianza APAIS con Unidad Primero en los cantones de la provincia de Manabí.

Tercero, estas alianzas electorales entre un partido nacional y un partido provincial para las elecciones provinciales y locales probaron ser una estrategia exitosa. Estas alianzas electorales ganaron en cinco provincias y en 34 cantones. En comparación, las alianzas electorales entre un partido nacional y un partido local ganaron en ocho cantones, entre un máximo de 12 cantones. Si se agrupa los votos obtenidos por los candidatos que compitieron en elecciones por una lista provincial, en alianzas electorales entre un partido nacional y un partido provincial, entre dos partidos provinciales o entre un partido provincial y un partido local, los candidatos provinciales alcanzaron aproximadamente dos millones de votos en todo el país, tanto en las elecciones provinciales como en las locales.

En términos relativos, los candidatos provinciales obtuvieron el $24,9 \%$ de los votos de los ecuatorianos para las elecciones de alcalde. Esto es menos que el desempeño electoral de APAIS (26,25\%), pero mayor a cualquier otro partido nacional. En este sentido, si los candidatos provinciales se consideran un conjunto, ellos bien pueden ser considerados como la segunda gran fuerza política del país. En suma, las elecciones locales y provinciales en Ecuador son en su mayoría dominadas por los partidos nacionales y provinciales y no por los partidos y candidatos locales. 


\section{Bibliografía}

Dandoy R. (2014) "Las elecciones locales y provinciales de 2014 en Ecuador”, Perspectivas, Mayo. Disponible en: http://library.fes.de/ pdf-files/bueros/quito/10774.pdf

Pachano S. (2004) "El territorio de los partidos. Ecuador, 1979-2002", in Kornblith Miriam, Mayorga René Antonio, Pachano Simon, Tanaka Martin, Ungar Bleier Elisabeth, Arturo Arévalo Carlos, Partidos politicos en la Región Andina: entre la crisis y el cambio, International IDEA, Stockholm , pp. 71-91.

Pachano S. (2007) "Partidos y sistema de partidos en el Ecuador", in Rafael Roncagliolo, Carlos Meléndez (eds.), La politica por dentro. Cambios y continuidades en las organizaciones politicas de los paises andinos, International IDEA, Stockholm, pp. 161-211.

Polga-Hecimovich J. (2013) "Overcoming the Regional Cleavage? The (Unprecedented) Territorial Nationalization of Alianza PAIS”, Working Paper 01, FLACSO-Quito Departamento de Estudios Políticos. 